\title{
Characterization of Zeno Behavior in Hybrid Systems Using Homological Methods
}

\author{
Aaron D. Ames and Shankar Sastry \\ Department of Electrical Engineering and Computer Sciences \\ University of California at Berkeley \\ Berkeley, CA 94720 \\ \{adames, sastry\}@eecs.berkeley.edu
}

\begin{abstract}
It is possible to associate to a hybrid system a single topological space-its underlying topological space. Simultaneously, every hybrid system has a graph as its indexing object-its underlying graph. Here we discuss the relationship between the underlying topological space of a hybrid system, its underlying graph and Zeno behavior. When each domain is contractible and the reset maps are homotopic to the identity map, the homology of the underlying topological space is isomorphic to the homology of the underlying graph; the nonexistence of Zeno is implied when the first homology is trivial. Moreover, the first homology is trivial when the null space of the incidence matrix is trivial. The result is an easy way to verify the nonexistence of Zeno behavior.
\end{abstract}

\section{INTRODUCTION}

A dynamical system is defined by both a topological space and a flow on that space, e.g., a manifold and a vector field on that manifold. Moreover, the topological space underlying the dynamical system gives information about the behavior of the flow on that space. A classic example of this is Morse theory which relates the homology of the topological space with the qualitative behavior of the dynamical system.

Similarly, a hybrid system can be viewed as a "space" $\mathbb{H}$, together with a collection of vector fields on that space. The question is: how does the homology of this "space" affect the behavior of the vector fields (and hence flows) on this space? In order to answer this question, among others, it was shown in [1] how to associate a singe topological space $\operatorname{Top}(\mathbb{H})$ to the "space" $\mathbb{H}$; this is referred to as the underlying topological space of the hybrid system $\mathbf{H}$. Considering the homology of this space, it is possible to define a homology theory for hybrid systems, termed hybrid homology and given by

$$
H H_{n}(\mathbb{H}, A):=H_{n}(\operatorname{Top}(\mathbb{H}), A),
$$

where $H_{n}(\operatorname{Top}(\mathbb{H}), A)$ is the homology of $\operatorname{Top}(\mathbb{H})$ with coefficients in an abelian group $A$.

The purpose of this paper is to further investigate the relationship between the homology of the underlying topological space of a hybrid system, i.e., hybrid homology, and the behavior of flows on the "space" $\mathbb{H}$. This is done by considering the underlying graph, $\Gamma$, of a hybrid system;

$\dagger$ This research is supported by the National Science Foundation (NSF award number CCR-0225610) this is the graph that every hybrid system has as its basic indexing object. Together with the results of [1], it is possible to show that when each of the domains of the hybrid system $\mathbf{H}$ are contractible and the reset maps are homotopic to the identity map, then

$$
H H_{n}(\mathbb{H}, \mathbb{R}) \cong H_{n}(\Gamma, \mathbb{R}),
$$

where $H_{n}(\Gamma, \mathbb{R})$ is the graph homology of $\Gamma$.

The relationship between the homology of the underlying topological space of the hybrid system and the homology of the underlying graph of the hybrid system is especially interesting because we will show in this paper that $H_{n}(\Gamma, \mathbb{R})$ dictates the type of behavior that the hybrid system can display. Namely, we will show that

$$
\begin{gathered}
\operatorname{dim}_{\mathbb{R}}\left(H H_{1}(\mathbb{H}, \mathbb{R})\right)=\operatorname{dim}_{\mathbb{R}}\left(H_{1}(\Gamma, \mathbb{R})\right)=0 \\
\Downarrow \\
\mathbf{H} \text { is not Zeno. }
\end{gathered}
$$

More generally, and with this result in hand, we define the distance of a hybrid system from being non-Zeno: $d(\mathbf{H}, \overline{\mathscr{Z}})$. It is possible to show that

$$
d(\mathbf{H}, \overline{\mathscr{Z}}) \leq \operatorname{dim}_{\mathbb{R}}\left(H H_{1}(\mathbb{H}, \mathbb{R})\right)=\operatorname{dim}_{\mathbb{R}}\left(H_{1}(\Gamma, \mathbb{R})\right) .
$$

Moreover, this condition is easy to verify since it will be seen that the homology of $\Gamma$ is determined by the incidence matrix of $\Gamma: K_{\Gamma}$. Namely, $\operatorname{dim}_{\mathbb{R}}\left(H_{1}(\Gamma, \mathbb{R})\right)=\operatorname{dim}_{\mathbb{R}}\left(K_{\Gamma}\right)$.

These relationships make some very deep statements about hybrid systems. With respect to the underlying topological space of a hybrid system, these relationships say that the topology of this space encodes some important and basic information about the behavior of the hybrid system. Moreover, they say that the homology of the underlying topological space of a hybrid system is almost never trivial and that, in the case when it is trivial, it excludes the most interesting behavior that hybrid systems display: Zenoness. This is very dissimilar to dynamical systems which almost always are assumed to have underlying topological spaces with trivial homology: $\mathbb{R}^{n}$. From this, a general thesis can be formed:

Thesis. Hybrid systems are fundamentally different from dynamical systems on $\mathbb{R}^{n}$, and this difference is encoded in the homology of their underlying topological spaces.

The results of this paper provide support for this statement. 


\section{HYBRID SYSTEMS}

In this section the definition of hybrid systems is reviewed (cf. [2],[3]) for the purpose of discussing Zeno behavior; in this definition there will be special attention given to the oriented graph underlying the hybrid system as this will be main focus of this paper.

\section{A. Basic Definitions}

We begin by introducing the definition of a hybrid system, follow with an explanation of the underlying graph of a hybrid system, and conclude with some examples.

Definition 2.1: Define a hybrid system as a tuple

$$
\mathbf{H}=(Q, E, D, G, R, X),
$$

where

- $Q=\{1, \ldots, m\} \subset \mathbb{Z}$ is a set of discrete states which is a finite subset of the integers.

- $E \subset Q \times Q$ is a set of edges that define relations between the domains. For $e=(i, j) \in E$, denote the source of $e$ by $\mathfrak{s}(e)=i$ and the target of $e$ by $\mathfrak{t}(e)=j$; sometimes the edges in $E$ will be indexed, i.e., we will label the edges so that $E=\left\{e_{1}, \ldots, e_{|E|}\right\}$ where $|E|$ is the cardinality of $E$.

- $D=\left\{D_{i}\right\}_{i \in Q}$ is a set of domains where $D_{i}$ is a subset of $\mathbb{R}^{n}$.

- $G=\left\{G_{e}\right\}_{e \in E}$ is a set of guards, where $G_{e} \subset D_{\mathfrak{s}(e)}$ is a set that determines the switching behavior of the hybrid system.

- $R=\left\{R_{e}\right\}_{e \in E}$ is a set of reset maps; these are continuous maps from $G_{e} \subseteq D_{\mathfrak{s}(e)}$ to $R_{e}\left(G_{e}\right) \subseteq$ $D_{t(e)}$.

- $X=\left\{X_{i}\right\}_{i \in Q}$ is a set of vector fields, such that $X_{i}$ is Lipschitz when restricted to $D_{i}$. The solution to $X_{i}$ with initial condition $x_{0} \in D_{i}$ is denoted by $\varphi_{t}^{i}\left(x_{0}\right)$.

2.1: The above definition has a $\operatorname{graph}^{1}$ as its basic indexing object, so the definition can be restated using an oriented graph. Let $\Gamma$ be an oriented (or directed) graph. This is a tuple $\Gamma=(Q, E)$ where $Q$ is a collection of vertices and $E$ is a collection of oriented (or directed) edges, i.e., $E \subset Q \times Q$ with a source $s(e)$ and a target $t(e)$. Comparing this definition with the definition above, a hybrid system is a tuple

$$
\mathbf{H}=(\Gamma, D, G, R, X) .
$$

The oriented graph $\Gamma$ is referred to as the graph underlying the hybrid system $\mathbf{H}$, or $\mathbf{H}$ has $\Gamma$ as its underlying graph. We typically will use a diagram to denote the graph $\Gamma$, wherein the source and target of each edge becomes clear. For example, if $\Gamma$ is given by a diagram of the form:

$$
j \stackrel{e_{1}}{\stackrel{e_{2}}{\longrightarrow}} k
$$

\footnotetext{
${ }^{1}$ Technically this is a pseudograph because loops and multiple edges
} between vertices are allowed.
Then from this diagram we can read of the definition of $\Gamma: Q=\{i, j, k\}$ and $E=\left\{e_{1}, e_{2}\right\}$ where $e_{1}=(i, j)$ and $e_{2}=(i, k)$.

Example 2.1 (Bouncing ball): The hybrid system simulating the behavior of a bouncing ball is the standard example of a hybrid system that displays Zeno behavior. The bouncing ball, as a hybrid system, will be denoted by $\mathbf{H}_{B}$. It has as its underlying graph $\Gamma_{B}$ given by the diagram

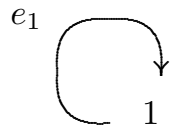

The other elements of the hybrid system are defined as: $D_{1}=\left\{\left(x_{1}, x_{2}\right): x_{1} \geq 0\right\}, G_{e_{1}}=\left\{\left(0, x_{2}\right): x_{2} \leq 0\right\}$, $X_{e_{1}}\left(x_{1}, x_{2}\right)=\left(x_{2},-g\right)$ and $R_{e_{1}}\left(x_{1}, x_{2}\right)=\left(0,-c x_{2}\right)$. Here $g$ is the acceleration due to gravity and $0<c<1$ is the amount of energy retained in each bounce.

Example 2.2 (The sign function): A classic example of a differential equation with a discontinuous right hand side is given by

$$
\dot{x}=\operatorname{sign}(x)=\left\{\begin{array}{ccc}
1 & \text { if } & x>0 \\
0 & \text { if } & x=0 \\
-1 & \text { if } & x<0
\end{array}\right.
$$

This can be formulated as a hybrid system $\mathbf{H}_{S}$. The underlying graph of $\mathbf{H}_{S}$ is given by $\Gamma_{S}$ which is defined by the diagram

$$
1 \underset{e_{2}}{\stackrel{e_{1}}{\longrightarrow}} 2
$$

The rest of the hybrid system is given by defining $D_{1}=$ $\{x \geq 0\}, D_{2}=\{x \leq 0\}, G_{e_{1}}=G_{e_{2}}=\{0\}, X_{1}(x)=1$ and $X_{2}(x)=-1$.

We also will consider the restricted sign function obtained by deleting an edge in $\Gamma_{S}$. We will denote the corresponding hybrid system by $\mathbf{H}_{S}^{-}$. More specifically, we define $\Gamma_{S}^{-}$by the diagram

$$
1 \stackrel{e_{1}}{\longrightarrow} 2
$$

and define $D_{1}, D_{2}, G_{e_{1}}, X_{1}$ and $X_{2}$ as before. Therefore, for $\mathbf{H}_{S}^{-}$we do not allow switching to occur in both directions.

\section{B. Zeno Behavior}

In order to discuss Zeno behavior in the context of hybrid systems, the definition of an execution must be introduced. Using the definition of an execution, two different types of Zeno executions-genuinely Zeno executions and chattering Zeno executions-can be introduced.

\section{2: A execution ${ }^{2}$ of the hybrid system $\mathbf{H}$ is a tuple}

$$
\varepsilon=(\tau, \xi, \eta),
$$

\footnotetext{
${ }^{2}$ Here we are considering only infinite executions since these are the executions that display Zeno behavior; introducing the definition of a finite execution would require unnecessary complication. For the more general definition see [2] or [3].
} 
where

- $\tau=\left\{\tau_{i}\right\}_{i \in \mathbb{N}}$ with $\tau_{0}=0 \leq \tau_{1} \leq \cdots \leq \tau_{j} \leq \cdots$ is a hybrid time sequence or a sequence of switching times.

- $\xi=\left\{\xi_{i}\right\}_{i \in \mathbb{N}}$ with $\xi_{i} \in \bigcup_{i \in Q} D_{i}$ is a sequence of initial conditions.

- $\eta=\left\{\eta_{i}\right\}_{i \in \mathbb{N}}$ with $\eta_{i} \in E$ is a hybrid edge sequence.

Additionally, we require that $\varepsilon=(\tau, \xi, \eta)$ must satisfy the condition that for $i \in \mathbb{N}$,

$$
\begin{aligned}
\tau_{i+1} & =\min \left\{t \geq \tau_{i}: \varphi_{t-\tau_{i}}^{\mathfrak{s}\left(\eta_{i}\right)}\left(\xi_{i}\right) \in G_{\eta_{i}}\right\} \\
\mathfrak{s}\left(\eta_{i+1}\right) & =\mathfrak{t}\left(\eta_{i}\right) \\
\xi_{i+1} & =R_{\eta_{i}}\left(\varphi_{\tau_{i+1}-\tau_{i}}^{\mathfrak{s}\left(\eta_{i}\right)}\left(\xi_{i}\right)\right) .
\end{aligned}
$$

We also require that $\varphi_{t-\tau_{i}}^{\mathfrak{s}\left(\eta_{i}\right)}\left(\xi_{i}\right) \in D_{\mathfrak{s}\left(\eta_{i}\right)}$ for all $t \in$ $\left[\tau_{i}, \tau_{i+1}\right]$.

Definition 2.2: A hybrid system $\mathbf{H}$ is Zeno if for some execution $\varepsilon$ of $\mathbf{H}$ there exists a finite constant $\tau_{\infty}$ such that

$$
\lim _{i \rightarrow \infty} \tau_{i}=\sum_{i=0}^{\infty}\left(\tau_{i+1}-\tau_{i}\right)=\tau_{\infty} .
$$

The execution $\varepsilon$ is called a Zeno execution.

2.3: The definition of a Zeno execution results in two qualitatively different types of Zeno behavior. They are defined as follows: for an execution $\varepsilon$ that is Zeno, $\varepsilon$ is

Chattering Zeno: If there exists a finite $C$ such that $\tau_{i+1}-\tau_{i}=0$ for all $i \geq C$.

Genuinely Zeno: If $\tau_{i+1}-\tau_{i}>0$ for all $i \in \mathbb{N}$.

The difference between these is prevalent especially in their detection and elimination. Chattering Zeno executions result from the existence of a switching surface in which the vector fields "oppose" each other; for this reason they are easy to detect. Fillipov solutions can be defined on these surfaces in order to force the flow to "slide" along the switching surface.

Genuinely Zeno executions are much more complicated in their behavior. There currently is no way to detect the existence of genuinely Zeno executions, and very little has been done in the area of eliminating these executions. This is due to the fact that genuinely Zeno executions are fundamentally global in nature, preventing the use of local techniques in their analysis.

Example 2.3: It is well known that the bouncing ball hybrid system $\mathbf{H}_{B}$ is genuinely Zeno; this can be verified easily by explicitly solving the vector fields. It is also well known that the sign function hybrid system $\mathbf{H}_{S}$ chatters; this is the quintessential example of chattering behavior. It can be verified that $\mathbf{H}_{S}^{-}$is not Zeno (by solving for the executions explicitly) and that $\mathbf{H}_{S}^{-}$and $\mathbf{H}_{S}$ have the same qualitative behavior with respect to the continuous variables.

\section{THE HOMOLOGY OF A GRAPH}

In this section, we will review the very basic elements of homology theory as well as the homology of a graph. If $\Gamma$ is an oriented graph, then we can associate to $\Gamma$ a chain complex; the homology of this chain complex is the homology of the graph (for a further review of these concepts, see [4]). We will restrict our attention to the homology of $\Gamma$ with coefficients in a field, specifically $\mathbb{R}$, because in this case the homology of $\Gamma$ is a vector space. The section will conclude by relating the incidence matrix with the homology of $\Gamma$. The motivation for considering the homology of a graph will become clear in the next section; the homology of the graph underlying a hybrid system is related (and in some cases isomorphic) to the homology of the underlying topological space of a hybrid system.

3.1: Let $R$ be a ring. A chain complex $C_{\bullet}$ is a family of $R$-modules, $\left\{C_{n}\right\}_{n \in \mathbb{Z}}$ together with $R$-module maps, called differentials, $d_{n}: C_{n} \rightarrow C_{n-1}$, i.e., it is a sequence

$$
\cdots C_{n+1} \stackrel{d_{n+1}}{\longrightarrow} C_{n} \stackrel{d_{n}}{\longrightarrow} C_{n-1} \cdots
$$

such that $d_{n} \circ d_{n+1}=0$. The $n^{\text {th }}$ homology of such a chain complex is denoted by $H_{n}(C)$ and is given by

$$
H_{n}\left(C_{\bullet}\right)=\frac{\operatorname{Ker}\left(d_{n}\right)}{\operatorname{Im}\left(d_{n+1}\right)} .
$$

We will be interested especially in the case when $C_{n}$ is a vector space over a field $\mathbb{F}$, and hence a $\mathbb{F}$-module. In this case we can consider the Euler characteristic of $C_{\bullet}$; this is given by

$$
\chi\left(C_{\bullet}\right)=\sum_{i \in \mathbb{Z}}(-1)^{i} \operatorname{dim}_{\mathbb{F}}\left(H_{i}\left(C_{\bullet}\right)\right),
$$

when the sum exists.

3.2: Given a graph $\Gamma$ and a field $\mathbb{F}$, we can associate to it a chain complex $C_{\bullet}(\Gamma, \mathbb{F})$ and hence we can consider its homology. First define

$$
C_{1}(\Gamma, \mathbb{F})=\bigoplus_{e \in E} \mathbb{F}=\mathbb{F}^{|E|}, \quad C_{0}(\Gamma, \mathbb{F})=\bigoplus_{i \in Q} \mathbb{F}=\mathbb{F}^{|Q|} .
$$

Let $\left\{\lambda_{e}\right\}_{e \in E}$ be a basis for $C_{1}(\Gamma, \mathbb{F})$ as a $\mathbb{F}$-module, i.e., every element $a \in C_{1}(\Gamma, \mathbb{F})$ can be written as $a=\sum_{e \in E} f_{e} \lambda_{e}$ for unique $f_{e} \in \mathbb{F}$. Similarly, let $\left\{\lambda_{i}\right\}_{i \in Q}$ be a basis for $C_{0}(\Gamma, \mathbb{F})$ as a $\mathbb{F}$-module, e.g., the standard basis for $\mathbb{F}^{|Q|}$.

With this in mind, define a map

$$
d=d_{1}: C_{1}(\Gamma, \mathbb{F})=\mathbb{F}^{|E|} \rightarrow C_{0}(\Gamma, \mathbb{F})=\mathbb{F}^{|Q|}
$$

by, for every $a \in C_{1}(\Gamma, \mathbb{F})$, setting

$$
d(a)=d\left(\sum_{e \in E} f_{e} \lambda_{e}\right):=\sum_{e \in E} f_{e}\left(\lambda_{\mathfrak{t}(e)}-\lambda_{\mathfrak{s}(e)}\right),
$$

for $f_{e} \in \mathbb{F}$.

By setting $C_{n}(\Gamma, \mathbb{F})=0$ for $n \neq 0,1$ and $d_{n}=0$ for $n \neq 1$, we complete our description of the chain complex 
associated to $\Gamma$, i.e., it is given by a sequence of differentials and vector spaces of the form:

$$
0 \stackrel{d_{2}=0}{\longrightarrow} C_{1}(\Gamma, \mathbb{F})=\mathbb{F}^{|E|} \stackrel{d=d_{1}}{\longrightarrow} C_{0}(\Gamma, \mathbb{F})=\mathbb{F}^{|Q|} \stackrel{d_{0}=0}{\longrightarrow} 0 .
$$

From this it follows that the homology of $\Gamma$ with coefficients in $\mathbb{F}$ is given by

$$
\begin{aligned}
& H_{1}(\Gamma, \mathbb{F})=\operatorname{Ker}(d) \\
& H_{0}(\Gamma, \mathbb{F})=\frac{\mathbb{F}^{|Q|}}{\operatorname{Im}(d)}=\operatorname{Coker}(d) .
\end{aligned}
$$

Using this we can consider the Euler characteristic of a graph $\Gamma$; it is given by

$$
\begin{aligned}
\chi(\Gamma) & =\operatorname{dim}_{\mathbb{F}}\left(H_{0}(\Gamma, \mathbb{F})\right)-\operatorname{dim}_{\mathbb{F}}\left(H_{1}(\Gamma, \mathbb{F})\right) \\
& =|Q|-\operatorname{dim}_{\mathbb{F}}(\operatorname{Im}(d))-\operatorname{dim}_{\mathbb{F}}(\operatorname{Ker}(d)) \\
& =|Q|-|E|,
\end{aligned}
$$

by the rank-nullity theorem. Note that we need not specify the coefficient field since the Euler characteristic is the same for any field. The Euler characteristic will prove useful for calculations.

Remark 3.1: The homology considered above is the homology of a graph with coefficients in a field; this is done because the homology of $\Gamma$ is then a vector space over $\mathbb{F}$, and we can consider its dimension $\operatorname{dim}_{\mathbb{F}}$. The homology of $\Gamma$ also can be considered over an arbitrary abelian group $A$, and is denoted by $H_{n}(\Gamma, A)$; in the case when $A=\mathbb{Z}$, it is just denoted by $H_{n}(\Gamma)$. Note that the homology of $\Gamma$ with coefficients in a field is related to the homology of $\Gamma$ with coefficients in $\mathbb{Z}$ by the universal coefficient theorem which states that

$$
H_{n}(\Gamma, \mathbb{F}) \cong H_{n}(\Gamma) \otimes_{\mathbb{Z}} \mathbb{F} \oplus \operatorname{Tor}_{1}^{\mathbb{Z}}\left(H_{n-1}(\Gamma), \mathbb{F}\right) .
$$

We now restrict our attention to the case when $\mathbb{F}=$ $\mathbb{R}$. This is done mainly for the sake of simplicity; the calculations that follow, for the most part, could be carried out with general fields and, in fact, general $R$-modules. This would involve more laborious proofs in some cases, and is unnecessary because no relevant information would be gathered. There are some cases in which considering the field $\mathbb{R}$ results in simplifications that otherwise might not be obtained. For example, because $\mathbb{R}$ is torsion free, the universal coefficient theorem implies that

$$
H_{n}(\Gamma, \mathbb{R}) \cong H_{n}(\Gamma) \otimes_{\mathbb{Z}} \mathbb{R},
$$

which is not true for a general field.

Definition 3.1: For the graph $\Gamma$, the incidence matrix, denoted by $K$, is a $|Q| \times|E|$ matrix given by

$$
K=\left(\begin{array}{lll}
\lambda_{\mathfrak{t}\left(e_{1}\right)}-\lambda_{\mathfrak{s}\left(e_{1}\right)} & \cdots & \lambda_{\mathfrak{t}\left(e_{|E|}\right)}-\lambda_{\mathfrak{s}\left(e_{|E|}\right)}
\end{array}\right),
$$

where $E=\left\{e_{1}, \ldots, e_{|E|}\right\}$ and $\lambda_{i}$ is the $i^{\text {th }}$ standard basis vector for $\mathbb{R}^{|Q|}$.
Proposition 3.1: Let $\mathscr{N}(K)$ be the null space of $K$ and let $\mathscr{R}(K)$ be its range, then

$$
\begin{aligned}
H_{0}(\Gamma, \mathbb{R}) & \cong \mathbb{R}^{|Q|-\operatorname{dim} \mathscr{R}(K)} \\
& =\mathbb{R}^{|Q|-|E|+\operatorname{dim} \mathscr{N}(K)} \\
H_{1}(\Gamma, \mathbb{R}) & \cong \mathbb{R}^{\operatorname{dim} \mathscr{N}(K)} .
\end{aligned}
$$

Example 3.1: For the bouncing ball hybrid system $\mathbf{H}_{B}$, the incidence matrix is given by $K_{B}=(0)$. This implies that $H_{1}\left(\Gamma_{B}, \mathbb{R}\right) \cong H_{0}\left(\Gamma_{B}, \mathbb{R}\right) \cong \mathbb{R}$.

For the sign function hybrid system $\mathbf{H}_{S}$, the incidence matrix is given by

$$
K_{S}=\left(\begin{array}{cc}
-1 & 1 \\
1 & -1
\end{array}\right) .
$$

Therefore, $H_{1}\left(\Gamma_{S}, \mathbb{R}\right) \cong H_{0}\left(\Gamma_{S}, \mathbb{R}\right) \cong \mathbb{R}$. Similarly, for $H_{S}^{-}$, we have

$$
K_{S}^{-}=\left(\begin{array}{c}
1 \\
-1
\end{array}\right) .
$$

So $H_{1}\left(\Gamma_{S}^{-}, \mathbb{R}\right) \cong 0$ and $H_{0}\left(\Gamma_{S}^{-}, \mathbb{R}\right) \cong \mathbb{R}$.

\section{THE UNDERLYING TOPOLOGICAL SPACE OF A HYBRID SYSTEM}

A dynamical system is defined by both a topological space and a flow on that space, e.g., a manifold and a vector field on that manifold. Similarly, a hybrid system can be thought of as a "space" and a set of vector fields on that space. With this in mind, the hybrid space, or H-space, underlying a hybrid system is given by the tuple

$$
\mathbb{H}=(\Gamma, D, G, R) \text {. }
$$

The main problem is that it is not clear how to associate to this tuple an actual topological space-one that encodes the proper information about the system.

In [1], it was shown that to associate to $\mathbb{H}$ a single topological space it is necessary to define hybrid systems in a different, yet analogous, way in order to clarify the relationships between the underlying space of a hybrid system, its H-space, and the behavior of that hybrid system. One possible and promising way of doing this is to define an $\mathrm{H}$-space as a certain type of small category $\mathfrak{H}$ and a functor from that small category to the category of topological spaces: Top. It is not the goal of this paper to go in depth into this construction, but we can give an overview of its major points. First we will show how to construct $\mathfrak{H}$ (as a graph) from $\Gamma$.

4.1: Let $\mathbf{H}$ be a hybrid system and $\Gamma$ its underlying graph. Then to $\Gamma$ we associate a graph $\mathfrak{H}_{\Gamma}$ (this is a simple graph, not a pseudograph). We define $\mathfrak{H}_{\Gamma}$ by defining, for each diagram $i \stackrel{e}{\longrightarrow} j$ in $\Gamma$, a diagram

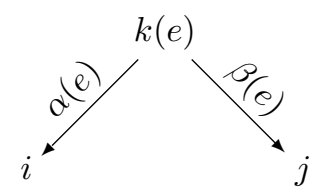


in $\mathfrak{H}$ such that $\left|Q_{\Gamma}\right|<k(e) \leq\left|Q_{\Gamma}\right|+\left|E_{\Gamma}\right|$ where $k(e)=$ $k\left(e^{\prime}\right)$ iff $e=e^{\prime}$.

We note that we can associate with $\mathfrak{H}_{\Gamma}$ a small category $\mathfrak{H}$ by defining its objects to be the vertices of this graph and its nonidentity morphisms to be the edges (of course, the identity morphism for each object also must be added to complete the definition). It is not possible to obtain a small category from $\Gamma$ in this way since the composition of two edges in $\Gamma$ would not necessarily be an edge in $\Gamma$.

Example 4.1: For the sign function hybrid system, $\mathfrak{H}_{\Gamma_{S}}$ is defined by the following diagram

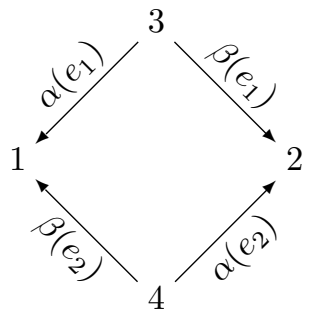

4.2: A categorical $\mathrm{H}$-space, $\mathbb{H}_{\mathfrak{C} \mathfrak{a t}}$, is a defined by

$$
\mathbb{H}_{\mathfrak{C a t}}=(\mathfrak{H}, \mathbf{S}),
$$

where $\mathfrak{H}$ is a specific type of small category (for example, as given in the above construction) called an $\mathrm{H}$-small category and $\mathbf{S}$ is a functor from $\mathfrak{H}$ to $\mathfrak{T o p}$. For the actual definitions of $\mathfrak{H}$ and $\mathbf{S}$ see [1]; space does not allow a review of all of these constructions. This paper emphasizes the relationship between the underlying graph $\Gamma$ of a hybrid system $\mathbf{H}$ and the small category $\mathfrak{H}$, which can be established without introducing the complete construction.

4.3: We can outline some of the important results that are obtained by considering categorical $\mathrm{H}$-spaces instead of $\mathrm{H}$-spaces (summarizing the results of [1]). The first necessary fact is that when $\mathfrak{H}$ is a $\mathrm{H}$-small category with a finite number of objects, there is a bijective correspondence:

$$
\{\mathrm{H}-\text { spaces, } \mathbb{H}\} \longleftrightarrow\left\{\text { categorical } \mathrm{H}-\text { spaces, } \mathbb{H}_{\mathfrak{C} \text { at }}\right\}
$$

and in fact there is an explicit way of constructing a categorical $\mathrm{H}$-space from an $\mathrm{H}$-space.

The second important fact is that by considering categorical $\mathrm{H}$-spaces as opposed to $\mathrm{H}$-spaces, it is possible to associate to $\mathbf{H}$ a single topological space, called the underlying topological space of $\mathbf{H}$, denoted by $\operatorname{Top}\left(\mathbb{H}_{\mathfrak{C} \mathfrak{a t}}\right)$ and given by the homotopy colimit:

$$
\operatorname{Top}\left(\mathbb{H}_{\mathfrak{C} \mathfrak{a t}}\right):=\operatorname{hocolim}^{\mathfrak{H}}(\mathbf{S}) .
$$

Given this, a homology theory for hybrid systems can be created, called hybrid homology, denoted by $H H_{n}\left(\mathbb{H}_{\mathfrak{C a t}}, A\right)$, and given by

$$
H H_{n}\left(\mathbb{H}_{\mathfrak{C a t}}, A\right):=H_{n}\left(\operatorname{Top}\left(\mathbb{H}_{\mathfrak{C a t}}\right), A\right),
$$

where $H_{n}\left(\operatorname{Top}\left(\mathbb{H}_{\mathfrak{C a t}}\right), A\right)$ is the homology of $\operatorname{Top}\left(\mathbb{H}_{\mathfrak{C a t}}\right)$ as a topological space with coefficients in an abelian group $A$. Fortunately, the hybrid homology can be computed very

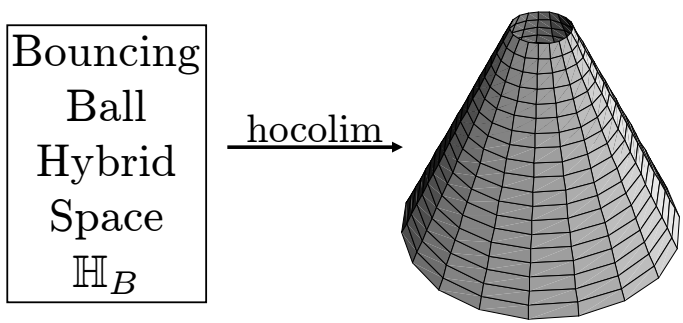

Fig. 1. The underlying topological space of the bouncing ball.

easily in a quite general case (which will be our focus here). When $\operatorname{Top}\left(\mathbb{H}_{\mathfrak{C} \mathfrak{a t}}\right)$ is domain contractible, i.e., when the domains $D_{i}$ are contractible for each $i \in Q$ and the reset maps $R_{e}$ are homotopic to the identity map for each $e \in E$,

$$
H H_{n}\left(\mathbb{H}_{\mathfrak{C a t}}, A\right) \cong H_{n}(\mathfrak{H}, A),
$$

where $H_{n}(\mathfrak{H}, A)$ is the homology of the small category $\mathfrak{H}$. The key point is that, in the case of an H-small category, the homology of the small category $\mathfrak{H}$ is equivalent to the homology of $\mathfrak{H}$ when it is viewed as a graph, i.e., it is the homology of $\mathfrak{H}_{\Gamma}$ as constructed above. Equally important is the fact that we will prove here: the graph homology of $\mathfrak{H}$-which is the graph homology of $\mathfrak{H}_{\Gamma}$-is isomorphic to the graph homology of $\Gamma$. This provides support for the thesis given in the introduction.

Theorem 1: If $\mathbf{H}$ is a hybrid system such that its underlying topological space, $\operatorname{Top}\left(\mathbb{H}_{\mathfrak{C a t}}\right)$, is domain contractible, then

$$
H H_{n}\left(\mathbb{H}_{\mathfrak{C a t}}, \mathbb{R}\right) \cong H_{n}(\mathfrak{H}, \mathbb{R}) \cong H_{n}\left(\mathfrak{H}_{\Gamma}, \mathbb{R}\right) \cong H_{n}(\Gamma, \mathbb{R}) .
$$

here $H_{i}(\mathfrak{H}, \mathbb{R})$ is the homology of the small category $\mathfrak{H}$ and $H_{n}\left(\mathfrak{H}_{\Gamma}, \mathbb{R}\right)$ is the homology of the graph $\mathfrak{H}_{\Gamma}$.

Example 4.2: For the bouncing ball hybrid system, $\mathbf{H}_{B}$, the underlying topological space is homotopic to the punctured cone (see Fig. 1). The hole in this cone is the warning that this hybrid system may be Zeno; it forces the first homology of this space to be nontrivial.

\section{A HOMOLOGY-BASED MEASURE OF ZENONESS}

Given a hybrid system $\mathbf{H}$, we can define a notion of how far that hybrid system is from being non-Zeno based on considering the graph $\Gamma$ underlying $\mathbf{H}$. To be more explicit, this distance can be defined to be the minimum number of edges of $\Gamma$ that need to be deleted such that the resulting hybrid system is not Zeno; in some sense this measures the likelihood that a Zeno execution will occur. We will make this definition more explicit, but first we need a little notation.

5.1: If $\widetilde{E} \subseteq E$, then define $\Gamma \backslash \widetilde{E}:=(Q, E \backslash \widetilde{E})$. Let $\mathbf{H}(\Gamma \backslash \widetilde{E})$ be the hybrid system with underlying graph 
$\Gamma \backslash \widetilde{E}$ obtained by setting $G(\Gamma \backslash \widetilde{E})=G \backslash\left\{G_{e}\right\}_{e \in \widetilde{E}}$ and $R(\Gamma \backslash \widetilde{E})=R \backslash\left\{R_{e}\right\}_{e \in \widetilde{E}}$.

Using this we can define the distance of $\mathbf{H}$ from being non-Zeno, $d(\mathbf{H}, \overline{\mathscr{Z}})$, by

$d(\mathbf{H}, \overline{\mathscr{Z}}):=\min \{|\widetilde{E}|: \widetilde{E} \subseteq E$ and $\mathbf{H}(\Gamma \backslash \widetilde{E})$ is not Zeno $\}$,

It is clear from this definition that $d(\mathbf{H}, \overline{\mathscr{Z}})=0$ implies that $\mathbf{H}$ is not Zeno because $\widetilde{E}=\emptyset$ is the empty set and $\mathbf{H}(\Gamma \backslash \emptyset)=\mathbf{H}$. Clearly, in order to define such a metric, we would need to have a necessary and sufficient condition for a hybrid system not to be Zeno. Since this presently is not available, the best we can hope for is to find bounds on $d(\mathbf{H}, \overline{\mathscr{Z}})$. In fact, giving a method for detecting the Zenoness or non-Zenoness of a hybrid system is equivalent to giving upper bounds on $d(\mathbf{H}, \overline{\mathscr{Z}})$; the tighter the bounds, the better the method. The key point is that just by considering the graph underlying the hybrid system, it is possible to obtain an upper bound on this distance.

5.2: A cycle, or circuit, in $\Gamma$ is a sequence of vertices $q_{1}, \ldots, q_{k} \in Q, q_{i} \neq q_{j}$, and edges between these vertices $e_{1}, \ldots, e_{k} \in E$ such that

$\left\{e_{1}\right\}=\left\{q_{1}, q_{2}\right\}, \quad\left\{e_{2}\right\}=\left\{q_{2}, q_{3}\right\}, \ldots,\left\{e_{k}\right\}=\left\{q_{k}, q_{1}\right\}$.

Here $\{e\}$ indicates that the orientation of the edge should be disregarded, i.e., if $e=\left(q_{i}, q_{j}\right),\{e\}=\left\{q_{i}, q_{j}\right\}=\left\{q_{j}, q_{i}\right\}$. Let $\Gamma_{\ell}$ denote the subgraph of $\Gamma$ formed by this cycle.

We can consider the set of all cycles defined by

$$
=\left\{\text { cycles } \Gamma_{\ell} \subseteq \Gamma\right\} .
$$

The important fact that will be needed about the homology of $\Gamma$ is its relationship to , which is the following:

$$
\operatorname{dim}_{\mathbb{R}}\left(H_{1}(\Gamma, \mathbb{R})\right)=||=\# \text { of cycles in } \Gamma .
$$

This fact was first discovered by Kirchhoff [5]. Morover, the number of Kirchhoff's independent current and voltage laws are $|Q|-\operatorname{dim}_{\mathbb{R}}\left(H_{0}(\Gamma, \mathbb{R})\right)$ and $\operatorname{dim}_{\mathbb{R}}\left(H_{1}(\Gamma, \mathbb{R})\right)$, respectively (cf. [6]). It is now possible to show the following proposition.

Proposition 5.1: Let $\Gamma$ be the graph underlying the hybrid system $\mathbf{H}$, then

$$
\begin{gathered}
\operatorname{dim}_{\mathbb{R}}\left(H_{1}(\Gamma, \mathbb{R})\right)=\operatorname{dim}_{\mathbb{R}}(\mathscr{N}(K))=0 \\
\Downarrow \\
\mathbf{H} \text { is not Zeno }
\end{gathered}
$$

where $K$ is the incidence matrix associated with $\Gamma$.

Using this we can establish the following theorem and its corollary.

Theorem 2: Let $\Gamma$ be the graph underlying the hybrid system $\mathbf{H}$, then

$$
d(\mathbf{H}, \overline{\mathscr{Z}}) \leq \operatorname{dim}_{\mathbb{R}}\left(H_{1}(\Gamma, \mathbb{R})\right)=\operatorname{dim}_{\mathbb{R}}(\mathscr{N}(K))
$$

where $K$ is the incidence matrix associated with $\Gamma$.

\section{Corollary 5.1: If $\Gamma$ is connected then}

$$
d(\mathbf{H}, \overline{\mathscr{Z}}) \leq 1-\chi(\Gamma)=1-|Q|+|E| .
$$

Example 5.1: Since $\operatorname{dim}\left(H_{1}\left(\Gamma_{B}, \mathbb{R}\right)\right)=1$, we cannot conclude that $\Gamma_{B}$ is not Zeno; this is a good thing since it is Zeno. Similarly, $\operatorname{dim}\left(H_{1}\left(\Gamma_{S}, \mathbb{R}\right)\right)=1$ so the same conclusion follows. We know that $d(\mathbf{H}, \overline{\mathscr{Z}})=1$, so it is possible to delete one edge and have a non-Zeno system. This is exactly the hybrid system $\mathbf{H}_{S}^{-}$; in this case it is possible to delete the edge and not change the qualitative behavior of the system. We also know that because $\operatorname{dim}\left(H_{1}\left(\Gamma_{S}^{-}, \mathbb{R}\right)\right)=0, \mathbf{H}_{S}^{-}$is not Zeno. A general way of determining when it is possible to remove edges from the underlying graph of the hybrid system in order to remove Zenoness, while simultaneously not changing the qualitative behavior of the hybrid system, is not known; this is the subject of further research.

\section{CONCLUSION}

In this paper, we have shown how to associate to every hybrid system-more specifically, to every hybrid space-a single topological space. Through homology, we were able to relate this space to the underlying graph of a hybrid system; since the homology of a graph is computable via its incidence matrix, this gives a concrete way of computing the homology of this space. The main result of this paper is that this homology and, more generally, this space gives useful information about the behavior of the hybrid system-especially with respect to Zeno. The distance of a hybrid system from being non-Zeno was defined, and we demonstrated that this distance is bounded above by the first homology of the underlying graph of a hybrid systemhence, the first homology of the underlying topological space of a hybrid system. Therefore, a hybrid system is not Zeno if the first homology of its underlying graph is trivial. This statement, coupled with the other results of this paper, supports the main thesis of this paper: the underlying topological space of a hybrid system is almost never trivial and, in the case when it is trivial, it excludes the most interesting behavior that hybrid systems display: Zenoness.

\section{REFERENCES}

[1] A. D. Ames and S. Sastry, "A homology theory for hybrid systems: Hybrid homology," in HSCC, ser. LNCS, M. Morari and L. Thiele, Eds., vol. 3414. Springer-Verlag, 2005, pp. 86-102.

[2] S. Simić, K. H. Johansson, S. Sastry, and J. Lygeros, "Towards a geometric theory of hybrid systems," in HSCC, ser. LNCS, B. Krogh and N. Lynch, Eds., vol. 1790. Springer Verlag, 2000, pp. 421-436.

[3] J. Zhang, K. H. Johansson, J. Lygeros, and S. Sastry, "Zeno hybrid systems," Int. J. Robust and Nonlinear Control, vol. 11, no. 2, pp. 435-451, 2001.

[4] C. A. Weibel, An Introduction to Homological Algebra. Cambridge University Press, 1994.

[5] G. Kirchhoff, "Über die auflösung der gleichungen, auf welche man bei der untersuchung der linearen verteilung galvanischer ströme gefürt wird," Ann. Phys. Chem, vol. 72, pp. 497-508, 1847.

[6] B. Andrásfai, Graph Theory: Flows, Matricies. Akadémiai Kiadó, 1991.

[7] N. Biggs, Algebraic Graph Theory, 2nd ed. Cambridge Mathematical Library, 1993. 